Matthew R. Smith and Philip Saylor, Massachusetts General Hospital Cancer Center, Boston, MA; Susan Halabi and Ben Sanford, Alliance Statistics and Data Center, Duke University Medical Center, Durham, NC; Charles J. Ryan and Eric J. Small, University of California at San Francisco, San Francisco, $\mathrm{CA}$; Walter Stadler, University of Chicago; Nirmala Bhoopalam, Loyola University of Chicago, Chicago, IL; Arif Hussain, University of Maryland, Baltimore, MD; Nicholas Vogelzang, Nevada Cancer Research Foundation CCOP Las Vegas, NV; Ralph J. Hauke, University of Nebraska, Omaha, NE; J. Paul Monk, Ohio State University, Columbus, OH; W. Kevin Kelly, Thomas Jefferson University, Philadelphia, PA; Michael Morris, Memorial SloanKettering Cancer Center, New York, NY; Fred Saad, Centre Hospitalier de I'Université de Montréal, Montreal, Canada.

Published online ahead of print at www.jco.org on March 3, 2014

Support information appears at the end of this article.

The content of this manuscript is solely the responsibility of the authors and does not necessarily represent the official views of the National Cancer Institute.

Authors' disclosures of potential conflicts of interest and author contributions are found at the end of this article.

Clinical trial information: NCT00079001

Corresponding author: Matthew R Smith, MD, PhD, Massachusetts General Hospital Cancer Center-Yawkey 7030, 55 Fruit St, Boston, MA 02114: e-mail: smith.matthew@mgh harvard.edu

() 2014 by American Society of Clinical Oncology

0732-183X/14/3211w-1143w/ $\$ 20.00$ DOI: $10.1200 / J C 0.2013 .51 .6500$

\title{
Randomized Controlled Trial of Early Zoledronic Acid in Men With Castration-Sensitive Prostate Cancer and Bone Metastases: Results of CALGB 90202 (Alliance)
}

Matthew R. Smith, Susan Halabi, Charles J. Ryan, Arif Hussain, Nicholas Vogelzang, Walter Stadler, Ralph J. Hauke, J. Paul Monk, Philip Saylor, Nirmala Bhoopalam, Fred Saad, Ben Sanford, W. Kevin Kelly, Michael Morris, and Eric J. Small

See accompanying article on page 1107

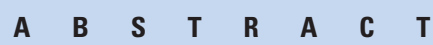

\section{Purpose}

Zoledronic acid decreases the risk for skeletal-related events (SREs) in men with castrationresistant prostate cancer and bone metastases but its role earlier in the natural history of the disease is unknown. This phase III study evaluated the efficacy and safety of earlier treatment with zoledronic acid in men with castration-sensitive metastatic prostate cancer.

\section{Patients and Methods}

Men with castration-sensitive prostate cancer and bone metastases whose androgen-deprivation therapy was initiated within 6 months of study entry were randomly assigned in a blinded 1:1 ratio to receive zoledronic acid (4 mg intravenously every 4 weeks) or a placebo. After their disease progressed to castration-resistant status, all patients received open-label treatment with zoledronic acid. The primary end point was time to first SRE, defined as radiation to bone, clinical fracture, spinal cord compression, surgery to bone, or death as a result of prostate cancer. Target accrual was 680 patients. Primary analysis was planned after 470 SREs. The study was discontinued prematurely (645 patients; 299 SREs) after the corporate supporter withdrew study drug supply.

\section{Results}

Early zoledronic acid was not associated with increased time to first SRE. The median time to first SRE was 31.9 months in the zoledronic acid group (95\% Cl, 24.2 to 40.3 ) and 29.8 months in the placebo group $(95 \% \mathrm{Cl}, 25.3$ to 37.2 ; hazard ratio, $0.97 ; 95 \% \mathrm{Cl}, 0$ to 1.17 ; one-sided stratified log-rank $P=$.39). Overall survival was similar between the groups (hazard ratio, $0.88 ; 95 \% \mathrm{Cl}, 0.70$ to $1.12 ; P=.29$ ). Rates of adverse events were similar between the groups.

\section{Conclusion}

In men with castration-sensitive prostate cancer and bone metastases, early treatment with zoledronic acid was not associated with lower risk for SREs.

\section{J Clin Oncol 32:1143-1150. (C) 2014 by American Society of Clinical Oncology}

\section{INTRODUCTION}

Bone metastases are an important cause of morbidity in men with prostate cancer. Most men with fatal prostate cancer develop bone metastases and bone is often the dominant or only site of metastatic disease. ${ }^{1}$ Bone metastases are associated with skeletalrelated events (SREs) including pathologic fractures, spinal cord compression, and need for surgery or radiation therapy to bone. ${ }^{2}$ Osteoclast-mediated bone destruction is the key pathologic mechanism for SREs in prostate cancer and other malignancies. ${ }^{3}$

Zoledronic acid is a bisphosphonate that potently inhibits osteoclast-mediated bone resorption.
Zoledronic acid is approved to treat men with prostate cancer, bone metastases, and disease progression despite androgen-deprivation therapy (ADT). In a randomized, placebo-controlled study of 643 men with castration-resistant prostate cancer (CRPC) and bone metastases, zoledronic acid was associated with a decreased rate of SREs at 15 months (44\% v 33\%; $P=.02)$ and increased time to first SRE (329 days $v>500$ days; $P=.011){ }^{4,5}$

Zoledronic acid is often used to treat men with castration-sensitive metastatic prostate cancer although its efficacy and safety in this setting is unknown. Characterizing potential benefits and harms of zoledronic acid in castration-sensitive disease is 
important because response duration for ADT is long and zoledronic acid has adverse effects (including renal impairment and osteonecrosis of the jaw) that appear related to cumulative drug exposure. ${ }^{6}$ For these reasons, we conducted a randomized controlled study to compare efficacy and safety of early administration of zoledronic acid in men with castration-sensitive disease versus standard zoledronic acid administration initiated after progression to castration-resistant disease. Patients with castration-sensitive prostate cancer and bone metastases were randomly assigned to either zoledronic acid (4 mg intravenously once every 4 weeks) or placebo. After their disease progressed to castration-resistant status, all patients received openlabel treatment with zoledronic acid. The primary study end point was time to first SRE, defined as radiation to bone, clinical fracture, spinal cord compression, surgery to bone, or death as a result of prostate cancer.

\section{PATIENTS AND METHODS}

\section{Patients}

Eligible patients were men at least 18 years old with histologically confirmed prostate adenocarcinoma, at least one bone metastasis by radiographic imaging (bone scan, magnetic resonance imaging, computed tomography, or plain radiographs), and Eastern Cooperative Oncology Group (ECOG) performance status 0 to 2 . All patients had creatinine clearance (calculated by Cockroft-Gault equation) of more than $30 \mathrm{~mL} / \mathrm{min}$. Patients received ADT (bilateral orchiectomies, gonadotropin-releasing hormone agonist, or gonadotropin-releasing hormone antagonist) for $\leq 6$ months before randomization and continued ADT throughout the study. Patients treated with intermittent ADT were not eligible, except for patients concurrently enrolled in Southwest Oncology Group 9346/INT-0162/Cancer and Leukemia Group B (CALGB) $9594^{7}$.

Key exclusion criteria included external-beam radiation therapy within 4 weeks, prior treatment with a bisphosphonate, or prior treatment with radiopharmaceuticals. Patients who received ADT at any time more than 6 months before enrollment were excluded, except for patients with prior neoadjuvant/ adjuvant $\mathrm{ADT}$ as long as that therapy was $\leq 6$ months duration and was completed more than 6 months before study entry. Patients with corrected serum calcium less than $8.0 \mathrm{mg} / \mathrm{dL}(2.00 \mathrm{mmol} / \mathrm{L})$ or $\geq 11.6 \mathrm{mg} / \mathrm{dL}(2.90$ $\mathrm{mmol} / \mathrm{L}$ ) were excluded.

The corporate sponsor provided study drug and financial support. The study was approved by the institutional review board or ethics committee for each site. All patients provided written informed consent.

\section{Treatment}

Patients were randomly assigned in a blinded 1:1 ratio to receive zoledronic acid intravenously or placebo (sterile saline) intravenously over a period of 15 minutes once every 4 weeks. Randomized block design was used. Randomization was stratified by ECOG performance status ( 0 to 1 or 2$)$, prior SRE (yes or no), and serum alkaline phosphatase ( $<$ upper limit of normal or $\geq$ upper limit of normal). For the patients with baseline creatinine clearance higher than $60 \mathrm{~mL} / \mathrm{min}$, zoledronic acid dose was $4.0 \mathrm{mg}$. For patients with creatinine clearances of 50 to $60 \mathrm{~mL} / \mathrm{min}, 40$ to $49 \mathrm{~mL} / \mathrm{min}$, and 30 to 39 $\mathrm{mL} / \mathrm{min}$, zoledronic acid doses were $3.5 \mathrm{mg}, 3.3 \mathrm{mg}$, and $3.0 \mathrm{mg}$, respectively. After determining the starting dose for each patient, there were no subsequent dose modifications. For patients with baseline serum creatinine less than 1.4 $\mathrm{mg} / \mathrm{dL}$ at study entry, an increase of $\geq 0.5 \mathrm{mg} / \mathrm{dL}$ required treatment delay until return of serum creatinine to $\leq 110 \%$ of baseline value. For patients with baseline serum creatinine levels $\geq 1.4 \mathrm{mg} / \mathrm{dL}$ at study entry, an increase of $\geq 1.0 \mathrm{mg} / \mathrm{dL}$ required treatment delay until return of serum creatinine to $\leq 110 \%$ of baseline value. Treatment delays of more than 4 weeks because of creatinine elevation required discontinuation of study treatment. The criteria for treatment delays owing to creatinine elevation were the same throughout the study.

Patients continued their assigned treatment until progressive disease, which was defined as new bone metastases or prostate-specific antigen (PSA) progression (defined as three consecutive rises in PSA with each PSA measurement at least 2 weeks apart and at least one PSA value $>4 \mathrm{ng} / \mathrm{mL}$ ). At progressive disease, patients began open-label treatment with zoledronic acid intravenously over 15 minutes every 3 weeks. The blind was maintained throughout the study. Patients continued study treatment until the first SRE.

Patients continued standard ADT throughout the study. Patients were instructed to take calcium supplements (500 mg daily) and vitamin D (400 to $500 \mathrm{IU})$.

Patients were evaluated at every treatment cycle (every 4 weeks during blinded treatment, and every 3 weeks during open-label treatment). Bone scans were obtained before patient registration; there we no required radiographic assessments on study. Serum PSA was measured at every cycle.

\section{End Points}

The primary efficacy end point was time to first SRE, defined as the interval between date of randomization to either radiation therapy to bone (including use of bone-targeted radiopharmaceuticals), clinical fracture, spinal cord compression, surgery to bone, or death as a result of prostate cancer. Because most patients will experience symptomatic skeletal progression before death, prostate cancer death was included in the definition of SREs to avoid potential under-reporting of SREs. Secondary end points were overall survival, progression-free survival (PFS), and safety. Progression-free survival was defined as the interval between date of randomization to first bone progression,

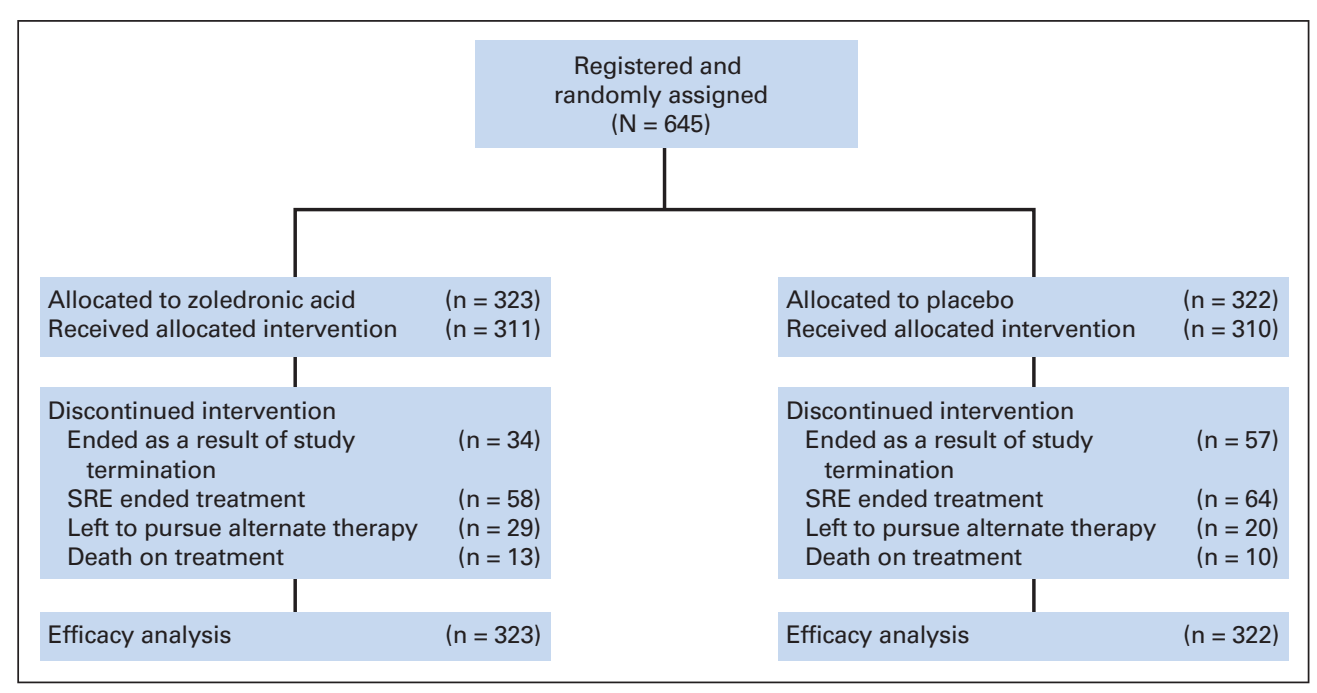

Fig 1. CONSORT diagram. SRE, skeletalrelated events. 
PSA progression, or death. Adverse events were graded using Common Terminology Criteria for Adverse Events version 3.

\section{Study Design and Data Analysis}

The primary end point was time to first SRE with a target sample size of 680. The null hypothesis was that the hazard ratio is greater than or equal to 1.0 versus the alternative hypothesis that the hazard ratio is less than 0.77 . With a target of 470 SREs, log-rank statistic had $88 \%$ power to detect a $23 \%$ decrease in hazard of SRE (equivalent to an increase in median time to SRE from 30 months to 39 months), assuming a one-sided type I error rate of .05. The following assumptions were made: accrual rate of 29 patients per month over

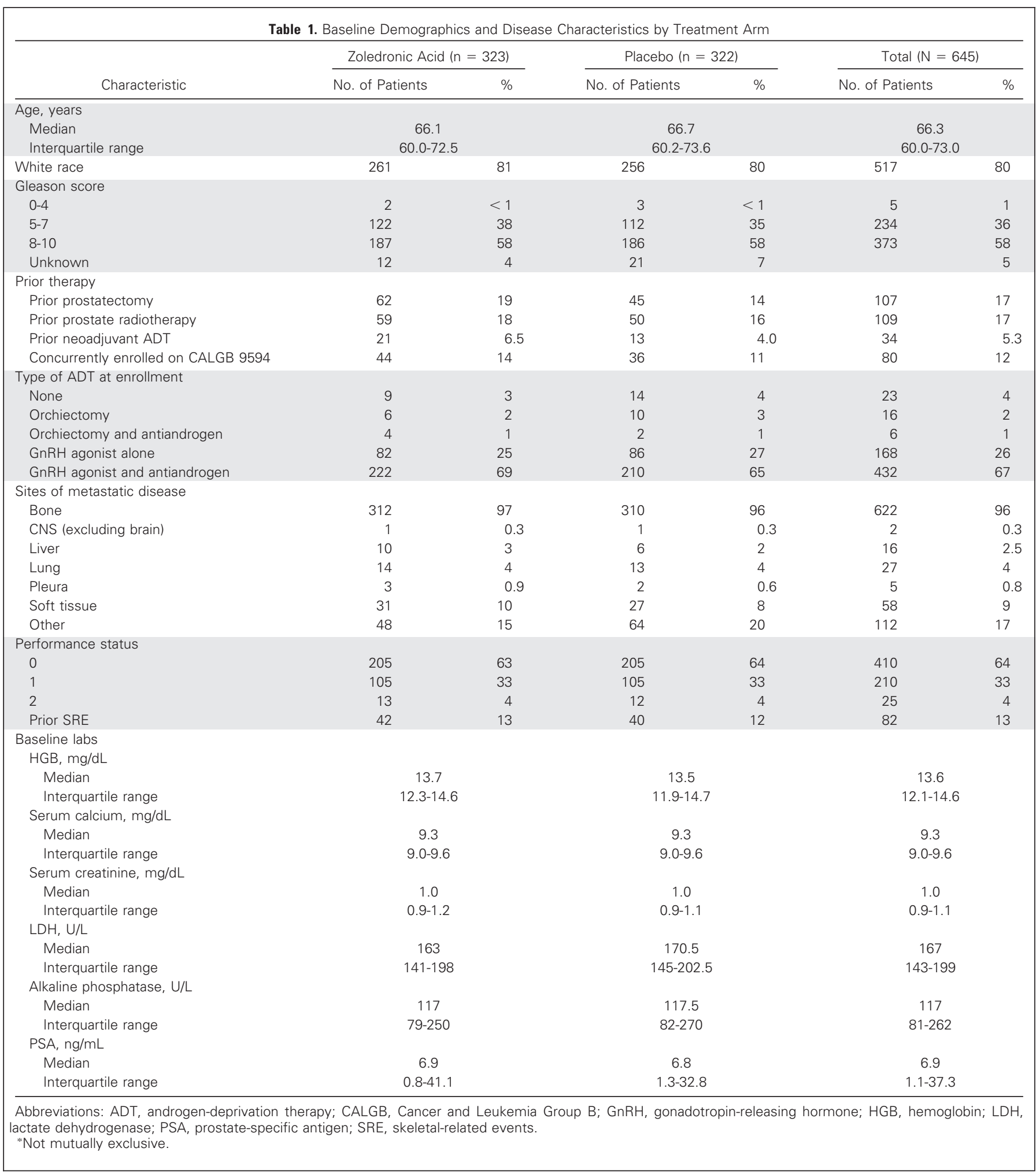


a 48-month enrollment period, a 36-month follow-up period after completion of study accrual, and exponential distribution for time to SRE.

This trial was monitored by the CALGB Data and Safety Monitoring board for efficacy and safety. Superiority and futility analysis were conducted for time to first SRE. Lan-Demets analog of the Emerson-Fleming sequential boundary was used to maintain overall significance level of $\alpha=.05$ while conducting interim analyses on time to first SRE. Final analysis was to be performed when 470 SRE events had been observed. Because of early termination, conditional power was performed under the alternative hypothesis. This is the probability that zoledronic acid is superior to placebo at the end of the trial, given time to first SRE data at interim analysis under alternative hypothesis.

An intention-to-treat approach was used in the analysis for all clinical end points, with the exception of toxicity. The primary analysis of time to first SRE end point was based on a one-sided stratified log-rank test for treatment effect, adjusting for stratification factors. The Kaplan-Meier product limit method was used to estimate time to first SRE, overall survival, and progression-free survival distributions. In addition, the proportional hazards model was used to test stratification factor by treatment interaction in predicting time to first SRE. All analyses were performed using SAS and R softwares, version 9.2. All $P$ values for secondary and exploratory analyses are two-sided.

The study was designed by the CALGB and approved by the Cancer Therapy Evaluation Program of the National Cancer Institute. The study was endorsed by ECOG, Southwest Oncology Group, and NCIC. The CALGB (Alliance) Statistics and Data Center performed registration, data collection, and statistical analyses.

As part of the CALGB quality assurance program, members of the audit committee visit all participating institutions at least once every 3 years to review source documents. Auditors verify compliance with federal regulations and protocol requirements, including those pertaining to eligibility, treatment, adverse events, tumor response, and outcome in a sample of protocols at each institution. Statistics and data center staff and the study chair reviewed the data; CALGB Audit Committee reviewed records onsite for 124 (19\%) of 645 enrolled patients.

\section{RESULTS}

\section{Study Population}

Between January 15, 2004 and May 31, 2012, 645 patients (323 in the zoledronic acid group and 322 in the placebo group) were randomly assigned (Fig 1). The study was closed to accrual on June 1, 2012 when the corporate supporter withdrew study drug supply. Based on the recommendation of the CALGB/Alliance Data and Safety Monitoring board, protocol treatment was terminated for all patients after July 2, 2012, and no further data were collected.

All randomly assigned patients were included in the efficacy analyses. Figure 1 illustrates patient disposition. All 621 patients who received at least one dose of study medication were included in the safety analyses. Forty-nine percent of the men in zoledronic acid group and $51 \%$ of men in the placebo group developed initiated open-label treatment with zoledronic acid. Median time on study was 11.8 months in the zoledronic acid group and 13.6 months in the placebo group.

Baseline demographics and disease characteristics were generally balanced between treatment groups (Table 1). Patients' median age was 66.1 years for the zoledronic acid group and 66.7 years for the placebo group. Nearly all patients in both groups had ECOG performance status 0 to 1 . Approximately $58 \%$ of patients in both groups had Gleason 8 to 10 tumors. Less than $10 \%$ of patients in both groups had received prior neoadjuvant ADT. Median PSA and alkaline phosphatase levels were balanced between the zoledronic acid and placebo groups.

\section{Efficacy}

As of March 25, 2013, there were 299 SREs (147 patients on the zoledronic acid group and 152 patients in the placebo group), which was $64 \%$ of the study target of 470 SRE events. Median times to first

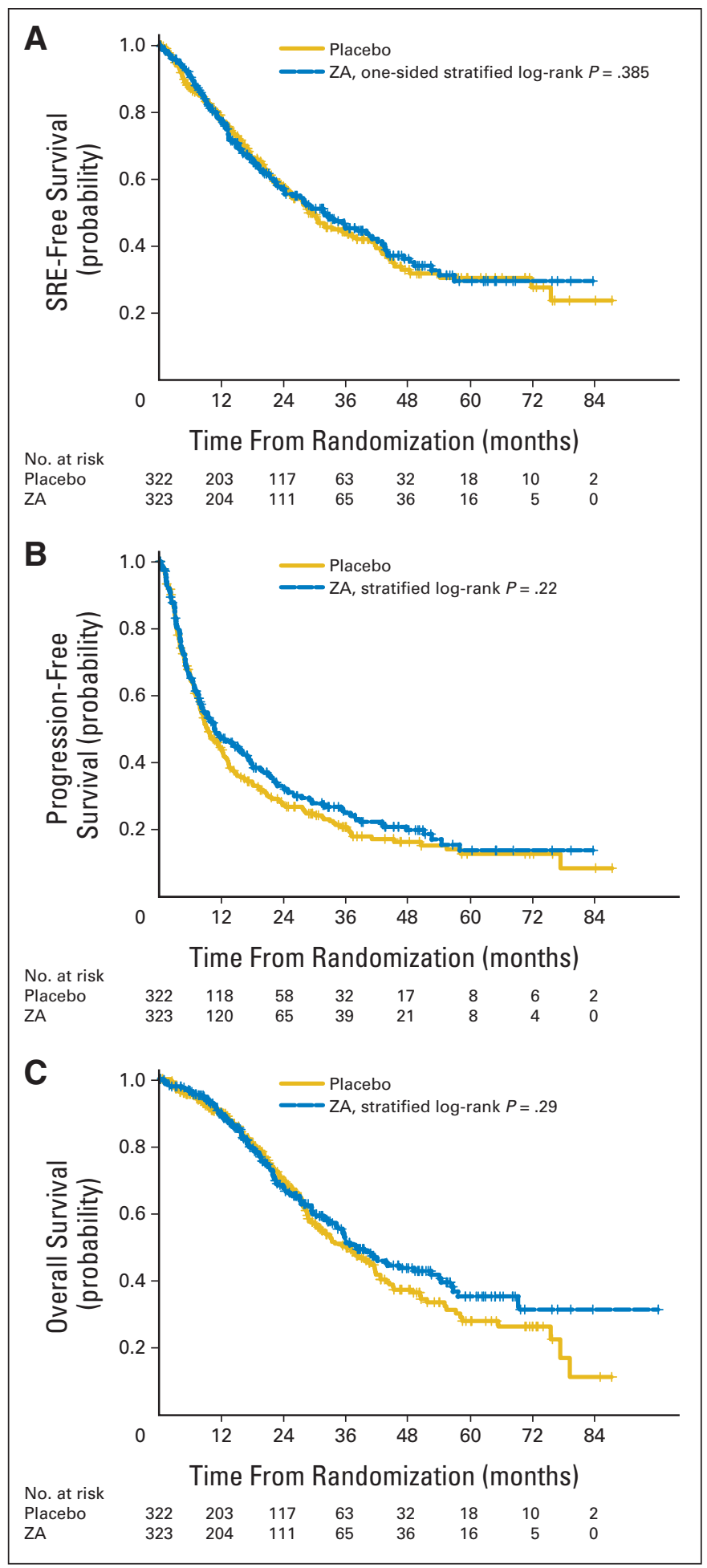

Fig 2. (A) Kaplan-Meier plot for skeletal-related events (SRE) -free survival by treatment arm. (B) Kaplan-Meier plot for progression-free survival by treatment arm. (C) Kaplan-Meier plot for overall survival by treatment arm. ZA, zoledronic acid. 
SRE were 31.9 (95\% CI, 24.2 to 40.3 ) and 28.8 months (95\% CI, 25.3 to 37.2 ) for the zoledronic acid and placebo groups, respectively (Fig 2A). There was no statistically significant difference in time to first SRE by treatment arm. Stratified hazard ratio (HR) was 0.97 (95\% CI, 0 to 1.174; one-sided stratified log-rank $P=.385$ ) for patients randomly assigned to zoledronic acid compared with patients assigned to placebo.

An exploratory subgroup analysis was performed based on prespecified stratification factors (Fig 3). Prior SRE by treatment interaction was almost statistically significant with time to first SRE $(P=.054)$. Among the subgroup of men with an SRE before study randomization $(\mathrm{n}=82)$, median time to first SRE was 31.9 months in the zoledronic acid group compared with 17.6 months in the placebo group (HR, 0.56; 95\% CI, 0.31 to 1.02 ). In the no-prior-SRE subgroup, median time to first SRE was similar for the zoledronic acid and placebo groups. Furthermore, there was no statistically significant interaction of stratification factor $(P=.139$ for performance status by treatment interaction; $P=.74$ for alkaline phosphatase by treatment interaction) by treatment for time to first SRE.

\section{Progression-Free Survival}

There were 441 PFS events (zoledronic acid group, $\mathrm{n}=211$; placebo group, $\mathrm{n}=230$ ). Median PFS times were 10.6 months (95\% CI, 8.5 to 15.4 ) and 9.2 months ( $95 \%$ CI, 8.0 to 11.9) for the zoledronic acid and placebo groups, respectively (Fig $2 \mathrm{~B}$ ). The majority of the events were PSA progression (70\%), followed by bone progression (22\%), and patient deaths (8\%). The adjusted HR was 0.89 (95\% CI, 0.74 to 1.07 ; stratified $\log$-rank $P=.22$ ) for patients randomly assigned to zoledronic acid compared with patients assigned to placebo.

\section{Overall Survival}

There were 285 patient deaths (zoledronic acid group, $n=134$; placebo group, $\mathrm{n}=151$ ). Median overall survival was 37.9 months
(95\% CI, 34.2 to 49.2 ) for the zoledronic acid group and 36.0 months (95\% CI, 30.2 to 41.6 ) for the placebo groups, respectively (Fig 2C). The adjusted HR is 0.88 (95\% CI, 0.70 to 1.12; stratified log-rank $P=$ .29) for zoledronic acid versus placebo group.

\section{Safety}

Adverse event data were reported for a total of 618 patients (96\%). Sixty-five patients in the zoledronic acid group and 38 patients in the placebo group withdrew from the study because of adverse events. The most common treatment-related grade 3 or higher events included pain (zoledronic acid group, 3\%; placebo group, 3\%), hypophosphatemia (zoledronic acid group, 3\%; placebo group, 2\%), fatigue (zoledronic acid, 3\%; placebo, 2\%), and hypocalcemia (zoledronic, 3\%; placebo, 1\%; Table 2). Two patients in each group discontinued study treatment because of creatinine elevation. One grade 5 event of renal failure was reported and attributed as possibly related to zoledronic acid treatment. Overall rates of grade 3 or higher treatment-related adverse events were approximately $14 \%$ in the zoledronic acid group (95\% CI, $11 \%$ to $19 \%$ ) and $12 \%$ in the placebo group (95\% CI, $8 \%$ to $16 \%)$. Ten zoledronic acid patients $(3.2 \%)$ and six placebo patients (1.9\%) experienced grade 3 osteonecrosis; all of these events were reported as treatment-related. Grade 3 or higher adverse events for creatinine elevation were reported for two patients in the zoledronic acid group and two patients in the placebo group.

\section{DISCUSSION}

In this randomized placebo-controlled trial of men with metastatic prostate cancer, early treatment with zoledronic acid for castrationsensitive disease was not associated with a decreased risk for SREs compared with treatment initiated after progression to castrationresistant disease. Overall survival and rates of adverse events were

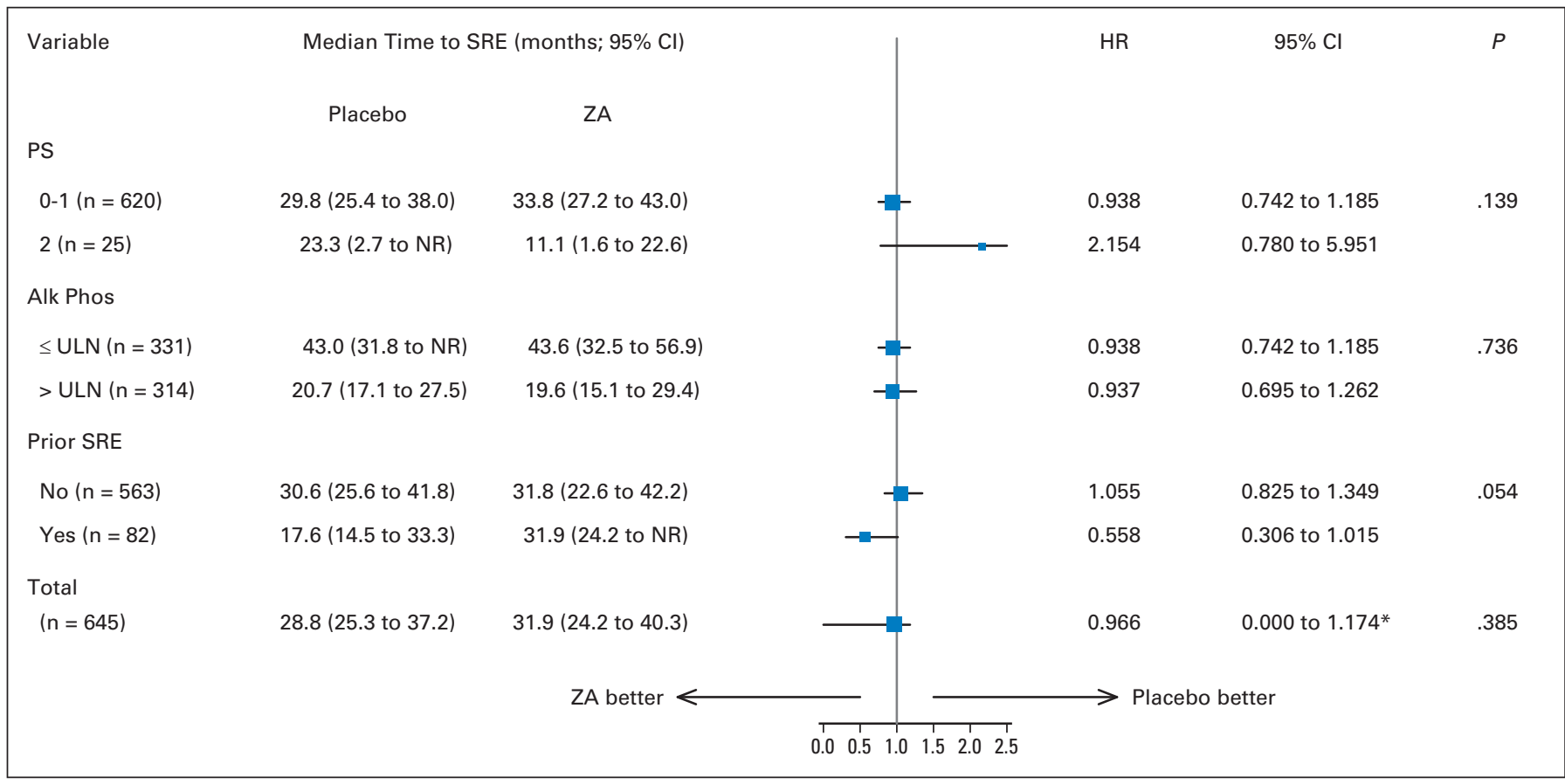

Fig 3. Forest plot of skeletal-related events (SRE) in select subgroups. $\left({ }^{*}\right)$ One-sided test. Alk Phos, alkaline phosphatase; HR, hazard ratio; NR, not reached; PS, performance status; ULN, upper limit of normal; ZA, zoledronic acid. 


\begin{tabular}{|c|c|c|c|c|c|c|c|c|c|c|}
\hline \multirow[b]{3}{*}{ Arm } & \multicolumn{4}{|c|}{ Grade of Adverse Event (CTCAE) } & & & & & & \\
\hline & \multicolumn{2}{|l|}{1 (mild) } & \multicolumn{2}{|c|}{2 (moderate) } & \multicolumn{2}{|l|}{3 (severe) } & \multicolumn{2}{|c|}{4 (life-threatening) } & \multicolumn{2}{|l|}{5 (lethal) } \\
\hline & No. of Patients & $\%$ & No. of Patients & $\%$ & No. of Patients & $\%$ & No. of Patients & $\%$ & No. of Patients & $\%$ \\
\hline \multicolumn{11}{|c|}{ Constitutional symptoms } \\
\hline \multicolumn{11}{|l|}{ Fatigue } \\
\hline ZA & 73 & 24 & 27 & 9 & 9 & 3 & 0 & 0 & 0 & 0 \\
\hline $\mathrm{Pl}$ & 63 & 21 & 36 & 12 & 6 & 2 & 1 & 0 & 0 & 0 \\
\hline \multicolumn{11}{|l|}{ Fever } \\
\hline ZA & 26 & 8 & 3 & 1 & 0 & 0 & 0 & 0 & 0 & 0 \\
\hline PI & 7 & 2 & 4 & 1 & 0 & 0 & 0 & 0 & 0 & 0 \\
\hline \multicolumn{11}{|l|}{ Rigors/chills } \\
\hline ZA & 21 & 7 & 0 & 0 & 0 & 0 & 0 & 0 & 0 & 0 \\
\hline PI & 10 & 3 & 2 & 1 & 0 & 0 & 0 & 0 & 0 & 0 \\
\hline \multicolumn{11}{|l|}{ Gastrointestinal } \\
\hline \multicolumn{11}{|l|}{ Anorexia } \\
\hline ZA & 19 & 6 & 5 & 2 & 1 & 0 & 0 & 0 & 0 & 0 \\
\hline $\mathrm{Pl}$ & 15 & 5 & 4 & 1 & 0 & 0 & 0 & 0 & 0 & 0 \\
\hline \multicolumn{11}{|l|}{ Diarrhea } \\
\hline ZA & 23 & 7 & 0 & 0 & 1 & 0 & 0 & 0 & 0 & 0 \\
\hline $\mathrm{Pl}$ & 11 & 4 & 0 & 0 & 0 & 0 & 0 & 0 & 0 & 0 \\
\hline \multicolumn{11}{|l|}{ Nausea } \\
\hline ZA & 30 & 10 & 1 & 0 & 0 & 0 & 0 & 0 & 0 & 0 \\
\hline $\mathrm{Pl}$ & 28 & 9 & 5 & 2 & 1 & 0 & 0 & 0 & 0 & 0 \\
\hline \multicolumn{11}{|c|}{ Metabolic/laboratory } \\
\hline \multicolumn{11}{|c|}{ Hypocalcemia } \\
\hline ZA & 35 & 11 & 6 & 2 & 5 & 2 & 2 & 1 & 0 & 0 \\
\hline $\mathrm{PI}$ & 42 & 14 & 9 & 3 & 2 & 1 & 1 & 0 & 0 & 0 \\
\hline \multicolumn{11}{|l|}{ Creatinine } \\
\hline ZA & 47 & 15 & 20 & 6 & 1 & 0 & 1 & 0 & 0 & 0 \\
\hline PI & 40 & 13 & 16 & 5 & 2 & 1 & 0 & 0 & 0 & 0 \\
\hline \multicolumn{11}{|c|}{ Hypomagnesemia } \\
\hline ZA & 14 & 5 & 0 & 0 & 0 & 0 & 0 & 0 & 0 & 0 \\
\hline $\mathrm{PI}$ & 20 & 7 & 1 & 0 & 0 & 0 & 0 & 0 & 0 & 0 \\
\hline \multicolumn{11}{|c|}{ Hypophosphatemia } \\
\hline ZA & 7 & 2 & 10 & 3 & 6 & 2 & 2 & 1 & 0 & 0 \\
\hline PI & 8 & 3 & 8 & 3 & 5 & 2 & 0 & 0 & 0 & 0 \\
\hline \multicolumn{11}{|l|}{ Hypokalemia } \\
\hline ZA & 21 & 7 & 1 & 0 & 2 & 1 & 0 & 0 & 0 & 0 \\
\hline $\mathrm{Pl}$ & 23 & 8 & 0 & 0 & 1 & 0 & 1 & 0 & 0 & 0 \\
\hline \multicolumn{11}{|l|}{ Pain } \\
\hline ZA & 58 & 19 & 29 & 9 & 9 & 3 & 0 & 0 & 0 & 0 \\
\hline $\mathrm{Pl}$ & 33 & 11 & 20 & 7 & 5 & 2 & 2 & 1 & 0 & 0 \\
\hline
\end{tabular}

similar between men assigned to early zoledronic acid and those assigned to treatment after progression to castration-resistant disease.

Only one other randomized controlled trial has evaluated the role of osteoclast-targeted therapy in men receiving initial ADT for metastatic prostate cancer. The Medical Research Council Pr05 study randomly assigned 311 men receiving primary ADT for metastatic prostate cancer to either clodronate (2,080 mg by mouth daily) or placebo. The primary study end point was symptomatic skeletal disease progression or death as a result of prostate cancer. After 59 months of follow-up, clodronate was associated with nonsignificant improvements in bone progression-free survival (HR, 0.79; 95\% CI, 0.61 to $1.02 ; P=.066$ ) and overall survival (HR, $0.80 ; 95 \% \mathrm{CI}, 0.62$ to $1.03 ; P=.082) .{ }^{8}$ Notably, a subsequent report with a longer follow-up period reported that clodronate was associated with greater overall survival, a secondary study end point. ${ }^{9}$

Our study has important strengths and limitations. Major strengths include the randomized placebo-controlled design and definition of SREs focused on the clinically relevant outcomes of radiation to bone, clinical fracture, spinal cord compression, surgery to bone, or death as a result of prostate cancer. The end point of our study is similar to the symptomatic skeletal event end point used in the pivotal study of radium-223 in men with castration-resistant prostate cancer and bone metastases. ${ }^{10}$ Although early termination limited the study power, the results seem to exclude any substantial benefit for early zoledronic acid on SRE risk in men with castration-sensitive disease. If the study was continued to completion (680 patients; 470 
events), our data indicate that the probability of correctly concluding that early zoledronic acid was superior to placebo is only $18 \%$ assuming a true hazard ratio of 0.77 .

In men with metastatic CRPC, prior SRE is associated with greater SRE risk. ${ }^{11}$ In an exploratory analysis of the current study, early zoledronic acid was associated with longer time to first on-study SRE in the small subgroup of men who experienced an SRE before study entry (HR, $0.56,95 \% \mathrm{CI}, 0.31$ to 1.02 ). Prospective validation would be necessary to establish whether early zoledronic acid improves outcomes for men with prior SRE or other high risk features.

SREs are a clinical manifestation of disease progression in bone. The results of our study suggest that ADT provides sufficient disease control to preclude any benefit from osteoclast-targeted therapy during the castration-sensitive disease state. Recent data from randomized controlled trials of other hormonal agents in metastatic CRPC support the concept that cancer control decreases SRE risk. In a randomized controlled trial of 1,199 men with metastatic CRPC and disease progression after docetaxel chemotherapy, enzalutamide was associated with improved overall survival and superiority over placebo for all secondary end points including time to first SRE (HR, 0.69; 95\% CI, 0.57 to $0.84 ; P<.001) .{ }^{12}$ In another randomized controlled trial of men with metastatic CRPC and disease progression after docetaxel chemotherapy, abiraterone acetate was associated with improved survival and lower rates of SREs. ${ }^{13}$ Additional studies are needed to determine whether these new agents supplant the need for osteoclasttargeted therapy in men with metastatic CRPC.

Denosumab is a fully human monoclonal antibody that specifically binds and inactivates receptor activator of NF- $\kappa$ B ligand, an essential mediator of osteoclast formation, function, and survival. In a global randomized controlled trial of men with CRPC and bone metastases, denosumab (120 mg subcutaneously every 4 weeks) was superior to zoledronic acid for prevention of SREs. ${ }^{14}$ The superiority of denosumab to zoledronic in metastatic CRPC, however, does not provide evidence to support the administration of denosumab to prevent disease-related SREs in men with castration-sensitive prostate cancer. To date, no study has evaluated the efficacy of denosumab to prevent SREs in men with castration-sensitive metastatic prostate cancer.

ADT is associated with accelerated bone loss and greater fracture risk in men with prostate cancer. In men receiving ADT, intravenous zoledonic acid (4 mg annually) and oral alendronate (70 mg once weekly) have been shown to increase bone mineral density. ${ }^{15,16}$ Denosumab (60 mg once every 6 months) increases bone mineral density, decreases new vertebral fractures in men receiving ADT for prostate cancer, ${ }^{17}$ and is approved to prevent bone loss in this setting. The results of the current study do not diminish the importance of either evaluating fracture risk in pros- tate cancer survivors or drug therapy to prevent osteoporosis and fractures in appropriate individuals.

In summary, early treatment with zoledronic acid in men with castration-sensitive metastatic prostate cancer was not associated with decreased risk of SREs. These results do not support the routine use of zoledronic acid or other osteoclast-targeted therapies to prevent SREs in men with metastatic prostate cancer before progression to castration-resistant disease.

\section{AUTHORS' DISCLOSURES OF POTENTIAL CONFLICTS OF INTEREST}

Although all authors completed the disclosure declaration, the following author(s) and/or an author's immediate family member(s) indicated a financial or other interest that is relevant to the subject matter under consideration in this article. Certain relationships marked with a " $U$ " are those for which no compensation was received; those relationships marked with a "C" were compensated. For a detailed description of the disclosure categories, or for more information about ASCO's conflict of interest policy, please refer to the Author Disclosure Declaration and the Disclosures of Potential Conflicts of Interest section in Information for Contributors. Employment or Leadership Position: Nicholas Vogelzang, US Oncology Network (C) Consultant or Advisory Role: Nicholas Vogelzang, Novartis (C), Dendreon (C), Janssen Pharmaceuticals (C), Bayer HealthCare Pharmaceuticals (C), GlaxoSmithKline (C), Pfizer (C), Astellas Pharma/Medivation (C); Walter Stadler, Novartis (C); Fred Saad, Amgen (C), Novartis (C); Michael Morris, Millennium Pharmaceuticals (C), Bayer HealthCare Pharmaceuticals (U) Stock Ownership: None Honoraria: Nicholas Vogelzang, Physicians' Education Resource Research Funding: Fred Saad, Amgen, Novartis; Michael Morris, sanofi-aventis, Bayer HealthCare Pharmaceuticals, EXINI Diagnostics, Algeta Expert Testimony: None Patents: None Other Remuneration: None

\section{AUTHOR CONTRIBUTIONS}

Conception and design: Matthew R. Smith, Susan Halabi, W. Kevin Kelly, Eric J. Small

Administrative support: Eric J. Small

Provision of study materials or patients: Matthew R. Smith, Charles J. Ryan, Arif Hussain, Nicholas Vogelzang, Walter Stadler, Ralph J. Hauke, J. Paul Monk, Nirmala Bhoopalam, Fred Saad, Eric J. Small

Collection and assembly of data: Matthew R. Smith, Susan Halabi, Fred Saad, Ben Sanford

Data analysis and interpretation: Matthew R. Smith, Susan Halabi, Charles J. Ryan, Arif Hussain, Nicholas Vogelzang, Walter Stadler, Ralph J. Hauke, J. Paul Monk, Philip Saylor, Nirmala Bhoopalam, Ben Sanford, W. Kevin Kelly, Michael Morris, Eric J. Small

Manuscript writing: All authors

Final approval of manuscript: All authors

\section{REFERENCES}

1. Gralow JR, Biermann JS, Farooki $A$, et al: NCCN task force report: Bone health in cancer care. J Natl Compr Canc Netw 3:S1-S32, 2009 (suppl 7)

2. Saylor PJ, Lee RJ, Smith MR: Emerging therapies to prevent skeletal morbidity in men with prostate cancer. J Clin Oncol 29:3705-3714, 2011

3. Brown JE, Cook RJ, Major $P$, et al: Bone turnover markers as predictors of skeletal complica- tions in prostate cancer, lung cancer, and other solid tumors. J Natl Cancer Inst 97:59-69, 2005

4. Saad F, Gleason DM, Murray $R$, et al: A randomized, placebo-controlled trial of zoledronic acid in patients with hormone-refractory metastatic prostate carcinoma. J Natl Cancer Inst 94:14581468, 2002

5. Saad F, Gleason DM, Murray R, et al: Longterm efficacy of zoledronic acid for the prevention of skeletal complications in patients with metastatic hormone-refractory prostate cancer. J Natl Cancer Inst 96:879-882, 2004
6. Zometa (zoledronic acid) Injection [package insert]. East Hanover, NJ, Novartis Pharmaceutical Corporation, 2012

7. Hussain M, Tangen CM, Berry DL, et al: Intermittent versus continuous androgen deprivation in prostate cancer. N Engl J Med 368:13141325, 2013

8. Dearnaley DP, Sydes MR, Mason MD, et al: A double-blind, placebo-controlled, randomized trial of oral sodium clodronate for metastatic prostate cancer (MRC PR05 Trial). J Natl Cancer Inst 95:13001311, 2003 
9. Dearnaley DP, Mason MD, Parmar MK, et al: Adjuvant therapy with oral sodium clodronate in locally advanced and metastatic prostate cancer: Long-term overall survival results from the MRC PR04 and PR05 randomised controlled trials. Lancet Oncol 10:872-876, 2009

10. Parker C, Nilsson S, Heinrich D, et al: Alpha emitter radium-223 and survival in metastatic prostate cancer. N Engl J Med 369:213-223, 2013

11. Smith MR, Cook RJ, Coleman R, et al: Predictors of skeletal complications in men with hormone-refractory metastatic prostate cancer. Urology 70:315-319, 2007
12. Scher HI, Fizazi $K$, Saad $F$, et al: Increased survival with enzalutamide in prostate cancer after chemotherapy. N Engl J Med 367:1187-1197, 2012

13. de Bono JS, Logothetis CJ, Molina A, et al: Abiraterone and increased survival in metastatic prostate cancer. N Engl J Med 364:1995-2005, 2011

14. Fizazi $K$, Carducci M, Smith M, et al: Denosumab versus zoledronic acid for treatment of bone metastases in men with castration-resistant prostate cancer: A randomised, double-blind study. Lancet 377:813-822, 2011

15. Michaelson MD, Kaufman DS, Lee $H$, et al: Randomized controlled trial of annual zoledronic acid to prevent gonadotropin-releasing hormone agonistinduced bone loss in men with prostate cancer. $\mathrm{J}$ Clin Oncol 25:1038-1042, 2007

16. Greenspan SL, Nelson JB, Trump DL, et al: Effect of once-weekly oral alendronate on bone loss in men receiving androgen deprivation therapy for prostate cancer: A randomized trial. Ann Intern Med 146:416-424, 2007

17. Smith MR, Egerdie B, Hernández Toriz N, et al: Denosumab in men receiving androgendeprivation therapy for prostate cancer. $N$ Engl J Med 361:745-755, 2009

\section{Support}

Supported in part by Grants No. CA31946 and CA33601 from the National Cancer Institute to the Alliance for Clinical Trials in Oncology (Monica M. Bertagnolli, MD, Chair) and to the Alliance Statistics and Data Center (Daniel J. Sargent, PhD), respectively, as well as Novartis Oncology, Grant No. K24CA121990 (M.R.S), and research awards from the Prostate Cancer Foundation (M.R.S.).

\section{Gastrointestinal Cancers Symposium}

Save the date for the 2015 Gastrointestinal Cancers Symposium, scheduled for January 15-17, 2015, in San Francisco, $\mathrm{CA}$. This symposium is a specialized meeting designed to highlight the latest translational science and new approaches in diagnosis, treatment, and management of Gl cancers. Now in its 12th year, the Symposium continues to offer a fresh perspective on Gl cancers, with a special focus on the most pertinent information oncologists of all subspecialties need to know now to provide the highest quality of care. Meeting cosponsors include AGA, ASCO, ASTRO, and SSO.

For more information, visit gicasym.org.

Each year, ASCO, in conjunction with our cosponsors, organizes a wide array of high-quality meetings, providing educational and scientific programs to advance your understanding of cancer. Join us for one or more of ASCO's meetings to interact with oncology experts, network with colleagues, and earn CME credit. 


\section{Appendix}

The following institutions participated in this study: Christiana Care Health Services Community Clinical Oncology Program (CCOP), Wilmington, DE, Stephen Grubbs, MD (supported by CA45418); Dana-Farber Cancer Institute, Boston, MA, Harold J. Burstein, MD, PhD (supported by CA32291); Duke University Medical Center, Durham, NC, Jeffrey Crawford, MD (supported by CA47577); Georgetown University Medical Center, Washington, DC, Bruce Cheson, MD (supported by CA77597); Greenville CCOP, Cancer Center of Carolinas; Hematology-Oncology Associates of Central New York CCOP, Syracuse, NY, Jeffrey Kirshner, MD (supported by CA45389); Massachusetts General Hospital, Boston, MA, Jeffrey W. Clark, MD (supported by CA32291); Memorial Sloan-Kettering Cancer Center, New York, NY, Clifford A. Hudis, MD (supported by CA77651); Missouri Valley Consortium-CCOP, Omaha, NE, Gamini S. Soori, MD; Nevada Cancer Research Foundation CCOP, Las Vegas, NV, John A. Ellerton, MD (supported by CA35421); New Hampshire Oncology-Hematology PA, Concord, NH, Douglas J. Weckstein, MD; The Ohio State University Medical Center, Columbus, OH, Clara D. Bloomfield, MD (supported by CA77658); Roswell Park Cancer Institute, Buffalo, NY, Ellis Levine, MD (supported by CA59518); State University of New York Upstate Medical University, Syracuse, NY, Stephen L. Graziano, MD (supported by CA21060); University of California at San Francisco, San Francisco, CA, Charles J. Ryan, MD (supported by CA60138); University of Chicago, Chicago, IL, Hedy L. Kindler, MD (supported by CA41287); University of Illinois MBCCOP, Chicago, IL, David J. Peace, MD (supported by CA74811); University of Iowa, Iowa City, IA, Daniel A. Vaena, MD (supported by CA47642); University of Maryland Greenebaum Cancer Center, Baltimore, MD, Martin Edelman, MD (supported by CA31983); University of Nebraska Medical Center, Omaha, NE, Apar Ganti, MD (supported by CA77298); University of North Carolina at Chapel Hill, Chapel Hill, NC, Thomas C. Shea, MD (supported by CA47559); University of Vermont, Burlington, VT, Steven M. Grunberg, MD (supported by CA77406); Wake Forest University School of Medicine, Winston-Salem, NC, David D. Hurd, MD (supported by CA03927); Washington University School of Medicine, St Louis, MO, Nancy Bartlett, MD (supported by CA77440); Weill Medical College of Cornell University, New York, NY, John Leonard, MD (supported by CA07968); Yale University, New Haven, CT, Lyndsay N. Harris, MD (supported by CA16359 and CA86802). 